\title{
JURISPRUDENCIA AMBIENTAL DE LA UNIÓN EUROPEA (SEGUNDO SEMESTRE 2018)
}

\author{
ROSA GILES CARNERO \\ Profesora Titular de Derecho Internacional Público \\ Universidad de Huelva
}


Sumario: 1. Consideraciones introductorias. 2. Recursos por incumplimiento. 3. Recursos de anulación. 4. Cuestiones prejudiciales. 4.1. Régimen para el comercio de derechos de emisión de gases de efecto invernadero. 4.2. Conservación de los hábitats naturales y de la fauna y flora silvestres. 4.3. Evaluación ambiental. 4.4. Resíduos. 4.5. Fiscalidad y protección ambiental.

\section{CONSIDERACIONES INTRODUCTORIAS}

En el período comprendido entre el 1 de abril y el 30 de septiembre de 2018, resultan de interés para esta crónica veinte sentencias dictadas por el Tribunal de Justicia de la Unión Europea en asuntos relacionados con el derecho ambiental. En primer término, destaca en este periodo el número de sentencias que se produjeron como resultado de procedimientos incoados por la Comisión Europea en el marco del artículo 258 TFUE, en relación con el incumplimiento por parte de Estados miembros de actos legislativos de la Unión Europea que tienen por objeto la protección del medio ambiente. En un total de ocho sentencias, se abordó la responsabilidad de Alemania, Bulgaria, Italia, Malta y en dos casos de Polonia, por incumplimiento de diferentes normas sobre protección ambiental; así como la responsabilidad derivada del incumplimiento de sentencias anteriores en las que el Tribunal había fallado en contra de Eslovaquia, España e Italia. En todos los casos, el resultando de las sentencias fue la declaración de incumplimiento.

Asimismo, en el período examinado se dictaron dos sentencias en respuesta a recursos de anulación interpuestos conforme al artículo 263 TFUE, contra actos de las instituciones y órganos de la Unión Europea adoptados en aplicación de normativa con incidencia ambiental. El Tribunal General resolvió y estimó uno de estos recursos; mientras que el Tribunal de Justicia se pronunció sobre el segundo desestimando su pertinencia.

Por último, en el período incluido en la presente crónica se dictaron diez sentencias en respuesta a peticiones de decisión prejudicial remitidas al Tribunal de Justicia por órganos jurisdiccionales nacionales, en relación con la validez 0 la interpretación de distintas disposiciones de contenido ambiental del derecho de la Unión Europea. Las cuestiones abordadas en estos pronunciamientos son diversas, de forma que se han incluido en este apartado cinco epígrafes referidos a las diferentes materias abordadas en las sentencias. En el primer epígrafe aparecen dos sentencias relativas al régimen para el comercio de derechos de 
emisión de gases de efecto invernadero, materia que suele ser recurrente en la jurisprudencia del Tribunal; el segundo incluye cuatro sentencias relativas a la conservación de los hábitats naturales y de la fauna y flora silvestres; el tercer epígrafe introduce dos sentencias sobre evaluación ambiental; se añade un cuarto epígrafe en el que se incluye una sentencia relativa a residuos; y por último, aparece un apartado relativo a una sentencia en materia de fiscalidad con incidencia en la protección ambiental.

\section{RECURSOS POR INCUMPLIMIENTO}

En el periodo comprendido en la presente crónica, se dictaron ocho sentencias en respuesta a recursos por incumplimiento. En primer lugar y por orden cronológico, cabe citar la sentencia del Tribunal de Justicia de la Unión Europea (Gran Sala) de 17 de abril de 2018, as. C-441/17, Comisión Europea contra República de Polonia, en la que se declaró el incumplimiento por parte del Estado de diversos apartados de la Directiva 92/43/CEE, relativa a la conservación de los hábitats naturales y de la fauna y flora silvestres (Directiva Hábitats), en su versión modificada por la Directiva 2013/17/UE, relativa a la adhesión de la República de Croacia; y de la Directiva 2009/147/CE, relativa a la conservación de las aves silvestres (Directiva Aves), en su versión modificada por la Directiva $2013 / 17^{1}$.

El Tribunal declaró en este pronunciamiento el incumplimiento de diferentes apartados de la Directiva Hábitats. En primer lugar, señaló que se había vulnerado el artículo 6.3 al adoptarse un anexo al plan de gestión forestal del distrito de Białowieża, sin que se hubiera realizado una evaluación adecuada de las posibles repercusiones sobre la integridad del lugar de importancia comunitaria (Zona LIC) y de la zona de protección especial (Zona ZPE) Puszcza

\footnotetext{
${ }^{1}$ Directiva 92/43/CEE del Consejo, de 21 de mayo de 1992, relativa a la conservación de los hábitats naturales y de la fauna y flora silvestres, DO, L 206, de 22 de julio de 1992, p. 7; Directiva 2009/147/CE del Parlamento Europeo y del Consejo, de 30 de noviembre de 2009, relativa a la conservación de las aves silvestres, en su versión modificada por la Directiva 2013/17, DO, L 20, de 26 de enero de 2010, p. 7; y Directiva 2013/17/UE del Consejo, de 13 de mayo de 2013, por la que se adaptan determinadas directivas en el ámbito del medio ambiente, con motivo de la adhesión de la República de Croacia, DO, L 158, de 10 de junio de 2013, p. 193.
} 
Białowieska². En segundo lugar, el Tribunal analizó el posible incumplimiento del artículo 6.1 de la Directiva, en conjunción con el artículo 4, en sus apartados 1 y 2, de la Directiva Aves, debido a que no se establecieron las medidas de conservación necesarias para salvaguardar los hábitats y las especies que figuran en sus anexos, y las especies migratorias no contempladas en el anexo en las citadas Zonas LIC y ZPE. EI Tribunal constató que la ejecución de las operaciones de gestión forestal analizadas producirían la desaparición de una parte del lugar protegido e incluido en la Red Natura 2000, por lo que también declaró el incumplimiento de estos preceptos ${ }^{3}$. En tercer lugar, el Tribunal también observó la vulneración del artículo 12.1. a) y d) de la Directiva Hábitats al no garantizar la protección de varias especies de insectos incluidas en su anexo IV, ya que advirtió que las actividades controvertidas conllevaban la muerte de ejemplares, o la destrucción de sus lugares de reproducción y zonas de descanso 4 . Por último, el Tribunal declaró el incumplimiento del artículo 5.b) y d) de la Directiva Aves al no garantizar la protección de especies de aves incluidas en su artículo 1, ya que también en este aspecto constató que las actividades forestales conducirían al deterioro o a la destrucción de los lugares de reproducción o de las zonas de descanso de estas especies ${ }^{5}$.

El segundo pronunciamiento emitido en este periodo se incluyó en la sentencia del Tribunal de Justicia de la Unión Europea (Sala Segunda) de 26 de abril de 2018, as. C-97/17, Comisión Europea contra República de Bulgaria, en la que se declaró el incumplimiento de la Directiva 2009/147/CE. En este caso, el Tribunal consideró que Bulgaria había vulnerado el artículo 4.1 de la Directiva Aves al no incluir en las zonas de protección especial toda el área de la cordillera de Rila que era relevante para la protección de las especies incluidas en su anexo I. El Tribunal recordó la obligación de los Estados de establecer las clasificaciones generales o parciales de ZPE basándose en criterios ornitológicos científicamente fundados, de forma que analizó el caso teniendo en cuenta la

\footnotetext{
2 Véanse párrafos 127 y 151.

${ }^{3}$ Véase párrafo 218.

${ }^{4}$ Véase párrafo 236.

${ }^{5}$ Véase párrafo 259.
} 
información al respecto proporcionada por la Comisión Europea y el Estado ${ }^{6}$. A la vista de esta información, declaró que Bulgaria había incumplido su obligación de identificar los territorios adecuados para ser clasificados como ZPE, excediendo su margen de apreciación en este ámbito?

En tercer lugar, cabe reseñar la sentencia del Tribunal de Justicia de la Unión Europea (Sala Primera) de 31 de mayo de 2018, as. C-251/17, Comisión Europea contra República Italiana, en la que se declaró el incumplimiento de la sentencia anterior del Tribunal de Justicia de la Unión Europea (Sala Séptima) de 19 de julio de 2012, as. C-565/10, Comisión Europea contra República Italiana. En el primer pronunciamiento, se había declarado el incumplimiento por parte de Italia de la Directiva 91/271/CEE, relativa al tratamiento de las aguas residuales urbanas, en su versión modificada por el Reglamento 1137/2008, en lo que se refería, según los casos, al tratamiento de aguas residuales o el funcionamiento de estaciones de depuración de 109 aglomeraciones situadas en territorio italiano ${ }^{8}$. En este nuevo pronunciamiento, el Tribunal constató que persistía el incumplimiento en relación a diferentes aglomeraciones, por lo que procedió a determinar una sanción pecuniaria ${ }^{9}$. Para fijar la suma a pagar, el Tribunal tuvo en cuenta que la medida debía hacerse con el objetivo de apremiar al Estado al cumplimiento de la sentencia de 2012, y ateniendo en a la gravedad de las circunstancias del caso en relación al deterioro ambiental. En consecuencia, fijó tanto una multa coercitiva de 30.112 .500 euros a abonar cada semestre que no se hubiera dado cumplimiento a la sentencia, como una suma a tanto alzado de 25 millones de euros ${ }^{10}$.

Una nueva condena a Polonia se incluyó en la sentencia del Tribunal de Justicia de la Unión Europea (Sala Primera) de 31 de mayo de 2018, as. C-526/16,

\footnotetext{
${ }^{6}$ Véanse párrafos 61 y 62.

${ }^{7}$ Véase párrafo 65.

${ }^{8}$ Directiva 91 /271 /CEE del Consejo, de 21 de mayo de 1991, sobre el tratamiento de las aguas residuales urbanas, DO, L 135, de 30 de mayo de 1991, p.40; y Reglamento (CE) no 1137/2008 del Parlamento Europeo y del Consejo, de 22 de octubre de 2008 , por el que se adaptan a la Decisión 1999/468/CE del Consejo determinados actos sujetos al procedimiento establecido en el artículo 251 del Tratado, en lo que se refiere al procedimiento de reglamentación con control, DO, L 311, de 21 de noviembre de 2008, p.1.

${ }^{9}$ Véanse párrafos 34 a 40.

${ }^{10}$ Véanse párrafos $67,69,72$ y 98.
} 
Comisión Europea contra República de Polonia, en la que se declaró el incumplimiento de la Directiva 2011/92/UE, relativa a evaluación de impacto ambiental ${ }^{11}$. En su pronunciamiento, el Tribunal consideró que el Estado había vulnerado el artículo 2.1 en relación con el artículo 4, apartados 2 y 3, y los anexos II y III de esta Directiva "al haber excluido los proyectos de prospección o de exploración de yacimientos de gas de esquisto mediante perforaciones de una profundidad de hasta 5.000 metros del procedimiento por el que se determina si es necesaria una evaluación de impacto ambiental, a excepción de las perforaciones de una profundidad mínima de 1.000 metros en zonas de extracción de agua y de protección de masas de aguas interiores y en las zonas objeto de regímenes de protección de la naturaleza, como los parques nacionales, las reservas naturales, los parques naturales y las zonas "Natura 2000", y sus alrededores". Para llegar a esta conclusión, el Tribunal argumentó que, dadas las características de la prospección y explotación del gas de esquisto, debía suponerse, en principio, que suponían perforaciones profundas en el sentido del Anexo II de la Directiva 2011/92/UE y, por tanto y conforme al artículo 4.2 de este texto, debía determinarse caso a caso si el proyecto tiene que someterse a evaluación ${ }^{12}$.

El quinto pronunciamiento a incluir en esta crónica se refiere a la sentencia del Tribunal de Justicia de la Unión Europea (Sala Novena) de 21 de junio de 2018, as. C-543/16, Comisión Europea contra República Federal de Alemania, en la que se declaró el incumplimiento de la Directiva 91/676/CEE, relativa a la protección de las aguas contra la contaminación producida por nitratos utilizados en la agricultura, en su versión modificada por el Reglamento 1137/2008 ${ }^{13}$. En particular, el Tribunal señaló el incumplimiento del artículo 5, apartados 5 y 7 , en relación con los anexos II y III, al no haber adoptado medidas adicionales 0 acciones reforzadas tras constatar que el programa de acción relativo a la

\footnotetext{
${ }^{11}$ Directiva 2011/92/UE del Parlamento Europeo y del Consejo, de 13 de diciembre de 2011, relativa a la evaluación de las repercusiones de determinados proyectos públicos y privados sobre el medio ambiente, DO, L 26, de 28 de enero de 2012, p. 1.

${ }^{12}$ Véanse párrafos 57 y 58.

${ }^{13}$ Directiva 91/676/CEE del Consejo, de 12 de diciembre de 1991, relativa a la protección de las aguas contra la contaminación producida por nitratos utilizados en la agricultura, DO, L 375, de 31 de diciembre de 1991, p. 1.
} 
protección de las aguas contra este tipo de contaminación no era suficiente. A lo largo de la sentencia, se examinaron los diferentes informes disponibles para constatar que Alemania no había revisado su programa de acción, ni adoptado medidas suplementarias pese a haberse detectado las insuficiencias ${ }^{14}$. A lo que se añadió, de forma general, el recordatorio de que ante una Directiva como la evaluada, que contiene reglas especiales de protección ambiental, los Estados tienen la obligación de satisfacer plenamente la exigencia de seguridad jurídica a través de una legislación que asegure una trasposición clara y precisa ${ }^{15}$.

En sexto lugar, cabe señalar la sentencia del Tribunal de Justicia de la Unión Europea (Sala Tercera) de 21 de junio de 2018, as. C-557/15, Comisión Europea contra República de Malta, en la que se declaró el incumplimiento de la Directiva 2009/147/CE. El Tribunal consideró que se habían vulnerado las obligaciones de la Directiva Aves incluidas en los artículos 5. a) y e) y 8.1, en relación al artículo 9.1, al haberse adoptado un sistema de excepciones que permitía la captura de ejemplares vivos de siete especies de fringílidos silvestres. A lo largo de la sentencia, se analizó la fundamentación de las excepciones presentadas por el Estado a la luz de los datos proporcionados, y se desestimaron señalando la falta de una motivación que permitiera que fuera conforme con las previsiones de la Directiva ${ }^{16}$. Asimismo, se señaló que tampoco puede aceptarse que se cumplan los requisitos de pequeñas cantidades, captura selectiva, explotación prudente o aplicación en condiciones estrictamente controladas ${ }^{17}$.

El siguiente caso a reseñar en la presente crónica se incluyó en la sentencia del Tribunal de Justicia de la Unión Europea (Sala Cuarta) de 4 de julio de 2018, as. C-626/16, Comisión Europea contra República Eslovaca, en la que se declaró el incumplimiento de la sentencia anterior del Tribunal de Justicia de la Unión Europea (Sala Décima) de 25 de abril de 2013, as. C-331/11, Comisión Europea contra República Eslovaca. En el primer pronunciamiento, el Tribunal había declarado el incumplimiento por parte de Italia de la Directiva 1999/31/CE,

\footnotetext{
14 Véanse párrafos 71 y 120.

15 Véase párrafo 145.

${ }^{16}$ Véanse párrafos 51 y 52.

${ }^{17}$ Véanse párrafos 66, 86, y 97.
} 
relativa al vertido de residuos, al haberse autorizado la explotación del vertedero de Žilina - Považský Chlmec sin un plan de acondicionamiento adecuado ${ }^{18}$. En el nuevo pronunciamiento, se constató que el Estado no había adoptado las medidas necesarias para cumplir la sentencia, de forma que el vertedero seguía funcionando sin un plan de gestión adecuado, y en consecuencia, se procedió a fijar una sanción pecuniaria ${ }^{19}$. También en este caso el Tribunal consideró oportuno fijar tanto una multa coercitiva como una suma a tanto alzado. Para fijar la primera, se tuvo en cuenta la gravedad de la infracción, su duración y la capacidad de pago del Estado, de forma que de este análisis se derivó la condena a pagar 5.000 euros por cada día de retraso en la adopción de las medidas necesarias para dar cumplimiento a la sentencia de 2013, que comenzaría a contarse desde el pronunciamiento de la nueva sentencia ${ }^{20}$. Asimismo, el Tribunal condenó al Estado a pagar la suma a tanto alzado de 1.000.000 euros, como medida disuasoria para la repetición de infracciones similares ${ }^{21}$.

Un último caso que aborda el incumplimiento de un pronunciamiento anterior, se incluyó en la sentencia del Tribunal de Justicia de la Unión Europea (Sala Octava) de 25 de julio de 2018, as. C-205/17, Comisión Europea contra Reino de España, en la que se declaró el incumplimiento de la sentencia del Tribunal de Justicia de la Unión Europea (Sala Octava) de 14 de abril de 2011, as. C343/10, Comisión Europea contra Reino de España. En el primer pronunciamiento, el Tribunal había declarado el incumplimiento de la Directiva 1999/31/CE, relativa al tratamiento de aguas residuales y urbanas, en su versión modificada por el Reglamento (CE) n. $1137 / 20$, debido a no haber adoptado las medidas necesarias para la recogida de aguas residuales y el tratamiento de aguas residuales urbanas en diversas aglomeraciones de más de 15.000

\footnotetext{
${ }^{18}$ Directiva 1999/31/CE del Consejo, de 26 de abril de 1999, relativa al vertido de residuos, DO, L 182, de 16 de julio de 1999, p. 1.

${ }^{19}$ Véanse párrafos 55 y 59.

${ }^{20}$ Véanse párrafos 74,75, y 84 y siguientes.

${ }^{21}$ Véase párrafo 99.
} 
habitantes ${ }^{22}$. El Tribunal constató que persistía esta situación, y procedió a fijar una multa coercitiva y a una suma a tanto alzado $^{23}$. En lo que respecta a la multa coercitiva, el Tribunal volvió a subrayar el objetivo de persuasión para el cumplimiento del derecho de esta medida, de forma que en este caso señaló la oportunidad de establecer una multa decreciente en función de los progresos que fuera realizando el Estado ${ }^{24}$. En consecuencia, se condenó a España a abonar 10.950.000 euros por cada semestre de retraso en el cumplimiento de la sentencia 2011, teniendo en cuenta que esta cantidad se iría reduciendo en función de las aglomeraciones que obtuvieran el tratamiento adecuado. A esto se sumó la condena al pago de 12 millones de euros como suma a tanto alzado, calculada en función de la gravedad, la duración de la infracción y la capacidad de pago del Estado.

\section{RECURSOS DE ANULACIÓN}

En el período revisado en esta crónica, resultan relevantes dos sentencias emitidas en respuesta a sendos recursos de anulación presentados contra actos de la Unión Europea con contenido ambiental. En primer término y de nuevo siguiendo un orden cronológico, cabe señalar la sentencia del Tribunal General (Sala Primera Ampliada) de 17 de mayo de 2018, as. T-584/13, BASF Agro BV contra Comisión Europea, mediante la que se declaró la anulación de los artículos 1, 3 y 4 del Reglamento de Ejecución (UE) n.ำ 781/2013 de la Comisión, referido a las condiciones de aprobación de la sustancia activa fipronil, y a la prohibición del uso y la venta de semillas tratadas con productos fitosanitarios que contengan esta sustancia ${ }^{25}$. El Tribunal desarrolló en su pronunciamiento la reflexión sobre la aplicación del principio de cautela en este caso, y consideró que se había violado debido a que en el procedimiento la Comisión no había

\footnotetext{
22 Directiva 91/271/CEE del Consejo, de 21 de mayo de 1991, sobre el tratamiento de las aguas residuales urbanas, DO, L 135, de 30 de mayo de 1991, p. 40.

23 Véase párrafo 21.

${ }^{24}$ Véanse párrafos 65 y 66.

${ }^{25}$ Reglamento de Ejecución (UE) n. ${ }^{0} 781 / 2013$ de la Comisión, de 14 de agosto de 2013, por el que se modifica el Reglamento de Ejecución (UE) n. ${ }^{\circ}$ 540/2011 en lo relativo a las condiciones de aprobación de la sustancia activa fipronil, y se prohíben el uso y la venta de semillas tratadas con productos fitosanitarios que la contengan, DO, L. 219, de 15 de agosto de 2013, p. 22.
} 
desarrollado una evaluación de impacto ${ }^{26}$. Teniendo en cuenta que el Tribunal recordó la importancia de este principio en el marco de los procedimientos de autorización y aprobación para los productos fitosanitarios y sus sustancias activas, se procedió a la declaración de anulación de los preceptos señalados ${ }^{27}$.

En segundo lugar, mediante la sentencia del del Tribunal de Justicia (Sala Segunda) de 21 de junio de 2018, as. C-5/16, República de Polonia contra Parlamento Europeo y Consejo de la Unión Europea, se desestimó el recurso planteado contra la Decisión (UE) 2015/1814, relativa al establecimiento y funcionamiento de una reserva de estabilidad del mercado en el marco del régimen para el comercio de derechos de emisión de gases de efecto invernadero en la Unió ${ }^{28}$. El Tribunal desestimó todos los argumentos, destacando que el mecanismo previsto por la Decisión impugnada resultaba "adecuado para conseguir el objetivo de reducir la volatilidad del mercado de los derechos de emisión, sin ir más allá de lo necesario para lograrlo"29.

\section{CUESTIONES PREJUDICIALES}

\subsection{Régimen para el comercio de derechos de emisión de gases de efecto invernadero}

En el periodo comprendido en la presente crónica, el Tribunal de Justicia resolvió dos cuestiones prejudiciales planteadas en relación al régimen para el comercio de derechos de emisión de gases de efecto invernadero, un ámbito en el que suele ser habitual encontrar sentencias que aclaran la adecuación con la norma europea de diferentes aspectos de su aplicación. El primer pronunciamiento se incluyó en la Sentencia del Tribunal de Justicia (Sala Sexta) de 12 de abril de 2018, as. C-302/17, PPC Power a.s. contra Finančné riaditel'stvo Slovenskej

\footnotetext{
${ }^{26}$ Véase párrafo 173.

${ }^{27}$ Véase párrafo 57.

${ }^{28}$ Decisión (UE) 2015/1814 del Parlamento Europeo y del Consejo, de 6 de octubre de 2015, relativa al establecimiento y funcionamiento de una reserva de estabilidad del mercado en el marco del régimen para el comercio de derechos de emisión de gases de efecto invernadero en la Unión, y por la que se modifica la Directiva 2003/87/CE, DO, L. 264, de 9 de octubre de 2015, p. 1.

${ }^{29}$ Véase párrafo 175.
} 
republiky y Daňový úrad pre vybrané daňové subjekty, y resolvió una pregunta planteada por el Tribunal Regional de Bratislava (Eslovaquia) sobre la interpretación de la Directiva 2003/87/CE, por la que se establece un régimen para el comercio de derechos de emisión de gases de efecto invernadero en la Comunidad ${ }^{30}$.

Mediante esta cuestión prejudicial el órgano jurisdiccional nacional solicitó la interpretación del artículo 1 de esta disposición, en relación a la normativa nacional que gravaba al $80 \%$ de su valor los derechos de emisión de gases de efecto invernadero asignados gratuitamente que se hubieran vendido, o que no hubieran sido utilizados por las empresas ${ }^{31}$. El Tribunal recordó que la Directiva 2003/87 tiene como objetivo fomentar la reducción de las emisiones de gases de efecto invernadero "de una forma eficaz en relación con el coste y económicamente eficiente", y en relación con este objetivo consideró que el gravamen sobre el valor económico de los derechos de emisión fijado por un Estado miembro, no debería llevar a "disminuir el incentivo para reducir las emisiones de gases de efecto invernadero hasta el punto de suprimirlo por completo ${ }^{32}$. En el caso presentado, el gravamen suponía disminuir drásticamente el valor económico de los derechos de emisión, por lo que a juicio del Tribunal suprimía el incentivo que debería conllevar que las empresas pudieran tener un beneficio de la venta de sus derechos no utilizados ${ }^{33}$. En consecuencia, el Tribunal declaró que la interpretación de la Directiva 2003/87/CE se oponía a la normativa nacional controvertida.

Un segundo pronunciamiento en respuesta a una cuestión de contenido ambiental planteada se incluyó en la Sentencia del Tribunal de Justicia (Sala Sexta) de 17 de mayo de 2018, as. C-229/17, Evonik Degussa GmbH contra Bundesrepublik Deutschland, y resolvió la pregunta del Tribunal de lo

\footnotetext{
${ }^{30}$ Directiva 2003/87/CE del Parlamento Europeo y del Consejo, de 13 de octubre de 2003, por la que se establece un régimen para el comercio de derechos de emisión de gases de efecto invernadero en la Comunidad y por la que se modifica la Directiva 96/61/CE del Consejo, DO, L 275, de 25 de octubre de 2003 , p. 32.

${ }^{31}$ Véase párrafo 17.

${ }^{32}$ Véanse párrafos 18 y 15.

${ }^{33}$ Véase párrafo 27.
} 
Contencioso-Administrativo de Berlín (Alemania) sobre la interpretación de la Directiva 2003/87/CE, por la que se establece un régimen para el comercio de derechos de emisión de gases de efecto invernadero en la Comunidad; de la Decisión 2011/278/UE, por la que se determinan las normas transitorias de la Unión para la armonización de la asignación gratuita de derechos de emisión con arreglo al artículo 10 bis de la Directiva 2003/87; y de la Decisión 2013/448/UE, relativa a las medidas nacionales de aplicación para la asignación gratuita transitoria de derechos de emisión de gases de efecto invernadero con arreglo al artículo 11.3, de la Directiva 2003/87, en su versión modificada por la Decisión (UE) 2017/126 34 .

El litigio principal se había originado ante la negativa de la autoridad nacional competente a conceder una asignación gratuita de derechos de emisión de gases de efecto invernadero, por una actividad en la que se incluía un proceso de separación del hidrógeno de un flujo de gas enriquecido que ya contenía este elemento. El Tribunal consideró que la pregunta relevante en este caso era si un proceso que no permite generar hidrógeno mediante síntesis química, sino únicamente aislar el hidrógeno ya contenido en una mezcla gaseosa, queda dentro de los límites del sistema normativo señalado ${ }^{35}$. A este respecto, el Tribunal declaró que la interpretación del anexo I de la Decisión 2011/278/UE no permite incluir en el sistema de la referencia de producto para el hidrógeno a este tipo de proceso. A esto añadió la aclaración de que "para hallarse dentro de los límites del referido sistema sería necesario que ese proceso, por un lado, estuviera relacionado con la "producción de hidrógeno" en el sentido del anexo I de la Directiva 2003/87/CE [...], y, por otro, que guardara una relación de índole técnica con dicha actividad".

\footnotetext{
${ }^{34}$ Directiva 2003/87/CE del Parlamento Europeo y del Consejo, de 13 de octubre de 2003, por la que se establece un régimen para el comercio de derechos de emisión de gases de efecto invernadero en la Comunidad y por la que se modifica la Directiva 96/61/CE del Consejo, DO, L 275, de 25 de octubre de 2003, p. 32; Decisión 2011/278/UE de la Comisión, de 27 de abril de 2011, por la que se determinan las normas transitorias de la Unión para la armonización de la asignación gratuita de derechos de emisión con arreglo al artículo 10 bis de la Directiva 2003/87, DO, L 130, de 17 de mayo de 2011, p. 1; Decisión 2013/448/UE de la Comisión, de 5 de septiembre de 2013, relativa a las medidas nacionales de aplicación para la asignación gratuita transitoria de derechos de emisión de gases de efecto invernadero con arreglo al artículo 11, apartado 3, de la Directiva 2003/87, DO, L 240, de 7 de septiembre de 2013, p. 27; y Decisión (UE) 2017/126 de la Comisión, de 24 de enero de 2017, DO, L 19, de 25 de enero de 2017, p. 93.
}

${ }^{35}$ Véase párrafo 28. 


\subsection{Conservación de los hábitats naturales y de la fauna y flora silvestres}

En el período temporal fijado por esta crónica, el Tribunal de Justicia de la Unión Europea resolvió tres cuestiones prejudiciales relativas a la interpretación de normativa europea en materia de conservación de los hábitats naturales, y de la fauna y flora silvestres. Mediante la sentencia del Tribunal de Justicia de la Unión Europea (Sala Séptima) de 2 de abril de 2018, as. C-323/17, People Over Wind y Peter Sweetman contra Coillte Teoranta, se resolvió una pregunta planteada por el Tribunal Superior de Irlanda, sobre la interpretación de la Directiva 92/43/CEE, relativa a la conservación de los hábitats naturales y de la fauna y flora silvestres (Directiva Hábitats) ${ }^{36}$. En particular, en esta cuestión prejudicial se preguntó al Tribunal de Justicia sobre la interpretación de su artículo 6.3, relativo al mantenimiento, o el restablecimiento, en un estado de conservación favorable, de los hábitats naturales y de las zonas especiales de conservación, en relación a la instalación del cable de conexión a la red eléctrica de un parque eólico. En particular, el órgano jurisdiccional nacional preguntó si "para determinar si es o no necesario proceder posteriormente a una evaluación adecuada de las repercusiones de un proyecto sobre un lugar afectado, es posible, en la fase de evaluación previa, tener en cuenta las medidas encaminadas a evitar o a reducir los efectos perjudiciales de dicho proyecto sobre el lugar"37. La respuesta a esta pregunta sería la declaración del Tribunal de Justicia de que la interpretación del artículo 6.3 conlleva que "no procede, en la fase de evaluación previa, tener en cuenta las medidas encaminadas a evitar o a reducir los efectos perjudiciales de dicho plan o proyecto sobre este lugar".

En segundo lugar, la sentencia del Tribunal de Justicia de la Unión Europea (Sala Tercera) de 13 de junio de 2018, as. C-683/16, Deutscher Naturschutzring, Dachverband der deutschen Natur- und Umweltschutzverbände e.V. contra la República Federal de Alemania, resolvió una cuestión prejudicial planteada por el Tribunal de lo Contencioso-Administrativo de Colonia (Alemania), y que tuvo

\footnotetext{
${ }^{36}$ Directiva 92/43/CEE del Consejo, de 21 de mayo de 1992, relativa a la conservación de los hábitats naturales y de la fauna y flora silvestres, DO, L 206, de 22 de julio de 1992, p. 7.

${ }^{37}$ Véase párrafo 27.
} 


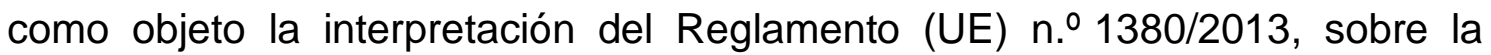
política pesquera común ${ }^{38}$. Se requirió, en este caso, la interpretación del artículo 11 de esta disposición, en relación a la negativa de las autoridades a establecer una prohibición de la pesca con artes de arrastre y redes de fondo en determinadas zonas marítimas.

El precepto referido se refiere a las medidas de conservación que los Estados miembros habrán de adoptar para el cumplimiento de sus obligaciones en virtud de la legislación medioambiental de la Unión, de forma que el Tribunal de Justicia analizó la situación controvertida en el litigio principal en relación al cumplimiento de las obligaciones que le incumben al Estado en el marco de la Directiva Hábitats y de la Directiva 2004/35/CE, sobre responsabilidad medioambiental en relación con la prevención y reparación de daños medioambientales ${ }^{39}$. Respecto a la primera y tras valorar los datos presentados por el órgano jurisdiccional nacional, el Tribunal declaró que el artículo 11 del Reglamento (UE) n. 0 1380/2013 "debe interpretarse en el sentido de que se opone a que un Estado miembro adopte las medidas aplicables a las aguas bajo su soberanía o jurisdicción que sean necesarias para cumplir las obligaciones que le incumben en virtud del artículo 6 de la Directiva 92/43/CEE del Consejo [...], y que prohíban con carácter general la pesca marítima profesional con artes de arrastre y redes de fondo en zonas Natura 2000, cuando tales medidas afecten a los buques de pesca que enarbolan el pabellón de otros Estados miembros"; mientras que en relación a la segunda, el mismo precepto "debe interpretarse en el sentido de que se opone a que un Estado miembro adopte medidas como las controvertidas en el litigio principal, aplicables a las aguas bajo su soberanía o jurisdicción, que sean necesarias para cumplir sus obligaciones derivadas de la Directiva 2004/35/CE [...].

\footnotetext{
${ }^{38}$ Reglamento (UE) n. ${ }^{1380 / 2013}$ del Parlamento Europeo y del Consejo, de 11 de diciembre de 2013,

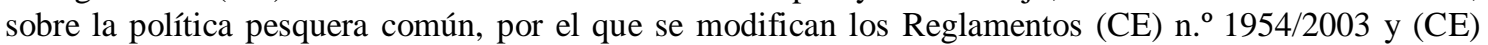
n. ${ }^{\circ}$ 1224/2009 del Consejo, y se derogan los Reglamentos (CE) n. ${ }^{\circ}$ 2371/2002 y (CE) n. ${ }^{\circ}$ 639/2004 del Consejo y la Decisión 2004/585/CE del Consejo, DO, L 354 de 28 de diciembre de 2013, p. 22.

39 Directiva 2004/35/CE del Parlamento Europeo y del Consejo, de 21 de abril de 2004, sobre responsabilidad medioambiental en relación con la prevención y reparación de daños medioambientales, DO, L 143 de 30 de abril de 2004, p. 56.
} 
Un tercer pronunciamiento a incluir en este apartado aparece en la sentencia del Tribunal de Justicia de la Unión Europea (Sala Segunda) de 25 de julio de 2018, as. C-164/17, Edel Grace y Peter Sweetman contra An Bord Pleanála, mediante la que se respondió a una pregunta planteada por el Tribunal Supremo de Irlanda, sobre la interpretación de la Directiva Hábitats. En particular, en este caso el órgano jurisdiccional nacional preguntó sobre la interpretación del artículo artículo 6, apartados 3 y 4, en relación a la autorización de un proyecto de parque eólico en el territorio de una zona de protección especial, clasificada así por albergar el hábitat natural de una especie protegida.

En el litigio principal se había constatado que el proyecto se realizaría en una zona de protección especial cuya superficie útil para la supervivencia de la especie protegida fluctuaba con el tiempo, y se cuestionaba si el proyecto era conforme a la Directiva Hábitat debido a que aunque conllevaría la intervención en el hábitat, incluirían medidas que evaluarían esta circunstancia y asegurarían el mantenimiento de la parte útil ${ }^{40}$. El Tribunal de Justicia tendría en cuenta el principio de cautela contenido en el artículo 6.3 de esta Directiva, y valoró la incertidumbre que presenta este tipo de actuaciones, de forma que declaró que en las circunstancias dadas las medidas dirigidas a proteger el hábitat tanto en el desarrollo como en el término del proyecto, no pueden tenerse en cuenta a efectos de su evaluación ambiental ${ }^{41}$. A esto añadió que las autoridades competentes podrían evaluar en un caso como éste que se dieran las circunstancias contempladas en el artículo 6.4, de forma que una vez que la evaluación se mostrara negativa, el proyecto pudiera autorizarse por razones imperiosas de interés público, teniendo en cuenta que en este caso tendrían que tomarse medidas compensatorias ${ }^{42}$.

El tercer pronunciamiento a incluir en este apartado apareció en la sentencia del Tribunal de Justicia de la Unión Europea (Gran Sala) de 25 de julio de 2018, as. C-528/16, Confédération paysanne y otros contra el Primer Ministro y el Ministerio de Agricultura, Agroalimentación y Foresta, mediante la que se respondió a una pregunta planteada por el Consejo de Estado francés, relativa

\footnotetext{
40 Véase párrafo 28.

${ }^{41}$ Véanse párrafos 52 a 54.

${ }^{42}$ Véanse párrafos 55 y 56.
} 
a la interpretación y la validez de la Directiva 2001/18/CE, sobre la liberación intencional en el medio ambiente de organismos modificados genéticamente, y la interpretación de la Directiva 2002/53/CE referente al catálogo común de las variedades de las especies de plantas agrícolas, en su versión modificada por el Reglamento (CE) n.ำ 1829/200343.

El litigio principal se refería a una normativa nacional que no consideraba que los organismos obtenidos mediante mutagénesis supusieran una modificación genética, de forma que permitía el cultivo y la comercialización de las variedades de colza obtenidas mediante esta técnica, y que eran tolerantes a los herbicidas. La primera pregunta que recibió el Tribunal de Justicia era si los organismos obtenidos mediante mutagénesis debían considerase organismos modificados genéticamente conforme a la Directiva 2001/18/CE. El Tribunal declaró que de la interpretación del artículo 2.2. de esta disposición, devenía una respuesta afirmativa $^{44}$.

El órgano jurisdiccional nacional había añadido una segunda cuestión a formular en el caso de que la anterior resultara positiva, de forma que cuestionaba si determinadas técnicas o métodos de mutagénesis podían excluirse del ámbito de aplicación de la Directiva. Para responder a esta segunda cuestión, el Tribunal procedió a la interpretación de las exenciones recogidas en el artículo 3.1 de la Directiva, en relación a su anexo I B y el considerando 17, utilizando el principio de cautela como primordial elemento de valoración ${ }^{45}$. En este sentido, el Tribunal declaró que el artículo 3.1 debe interpretarse en el sentido de que no excluye del ámbito de aplicación de la Directiva los organismos obtenidos mediante técnicas o métodos de mutagénesis como los controvertidos en el litio principal, sino únicamente aquellos "que han venido siendo utilizados convencionalmente en varios usos y para los que se dispone de una amplia experiencia de utilización segura". Asimismo, añadió que "debe interpretarse en

\footnotetext{
${ }^{43}$ Directiva 2001/18/CE del Parlamento Europeo y del Consejo, de 12 de marzo de 2001, sobre la liberación intencional en el medio ambiente de organismos modificados genéticamente y por la que se deroga la Directiva 90/220/CEE del Consejo, DO, L 106, de 17 de abril de 2001, p. 1; Directiva 2002/53/CE del Consejo, de 13 de junio de 2002, referente al catálogo común de las variedades de las especies de plantas agrícolas, DO, L 193, de 20 de julio de 2002, p. 1; y Reglamento (CE) n. ${ }^{\circ}$ 1829/2003 del Parlamento Europeo y del Consejo, de 22 de septiembre de 2003, DO, L 268, de 18 de octubre de 2003, p. 1.

${ }^{44}$ Véanse párrafos 35, 36 y 37.

${ }^{45}$ Véanse párrafos 50, 52 y 53.
} 
el sentido de que no tiene por efecto privar a los Estados miembros de la facultad de sujetar tales organismos a las obligaciones establecidas en dicha Directiva o a otras obligaciones, dentro del respeto del Derecho de la Unión, en particular de las normas relativas a la libre circulación de mercancías establecidas en los artículos 34 TFUE a 36 TFUE".

También se preguntó sobre si estos organismos debían considerarse exentos de las obligaciones recogidas en la Directiva 2002/53/CE, en su versión modificada por el Reglamento (CE) $n . \stackrel{0}{1829 / 2003}$. El Tribunal interpretó el artículo 4.4 de esta segunda disposición para declarar que debía darse esta exención únicamente respecto a "aquellas variedades obtenidas mediante técnicas o métodos de mutagénesis que han venido siendo utilizados convencionalmente en varios usos y para los que se dispone de una amplia experiencia de utilización segura", lo que no incluía las que eran objeto de controversia en el litigio principal $^{46}$.

\subsection{Evaluación ambiental}

En el período analizado en esta crónica, el Tribunal de Justicia emitió dos sentencias que respondía a cuestiones prejudicial relativas a la interpretación de normativa europea sobre evaluación ambiental. La primera de ella es la sentencia del Tribunal de Justicia de la Unión Europea (Sala Segunda) de 7 de junio de 2018, as. C-160/17, Raoul Thybaut y otros contra Región Valona, mediante la que se resolvió una cuestión prejudicial planteada por el Consejo de Estado de Bélgica que tuvo como objeto la interpretación de la Directiva 2001/42/CE, relativa a la evaluación de los efectos de determinados planes y programas en el medio ambiente ${ }^{47}$. En particular, el órgano jurisdiccional nacional presentó la cuestión sobre la interpretación del artículo 2.a) de esta disposición, en relación a la validez de un decreto del Gobierno de la Región

\footnotetext{
46 Véase párrafo 67.

${ }^{47}$ Directiva 2001/42/CE del Parlamento Europeo y del Consejo, de 27 de junio de 2001, relativa a la evaluación de los efectos de determinados planes y programas en el medio ambiente, DO, L 197, de 21 de julio de 2001, p. 30.
} 
Valona en el que se fijó un perímetro de reparcelación urbana relativo a una zona del municipio belga de Orp-Jauche.

La cuestión prejudicial planteada incidía en el concepto de "planes y programas" recogido en el artículo 2.a), y se refería a un litigio principal en el que se había cuestionado la determinación de un perímetro de reparcelación urbana que tenía como objeto establecer una zona en la que poder desarrollar la recalificación y el desarrollo de funciones urbanas, y que implicaba la actuación sobre vías terrestres y espacios públicos que tendrían que ser exonerados del cumplimiento de determinadas normas urbanísticas ${ }^{48}$. Para responder a esta petición, el Tribunal desarrolló su argumentación aludiendo al citado precepto, así como al artículo 3 de la Directiva referido a los actos que requieren una evaluación de impacto ambiental ${ }^{49}$. Conforme a este marco, tuvo en cuenta que la actuación controvertida requeriría un importante cambio normativo, y en aras del efecto útil de la Directiva declaró que debía interpretarse que la actuación de la Región de Valona estaba incluida en el "concepto de "planes o programas" que pueden tener efectos significativos en el medio ambiente en el sentido de dicha Directiva y tiene que ser objeto de una evaluación de los efectos medioambientales" 50 .

El segundo pronunciamiento a tener en cuenta en este apartado, es la sentencia del Tribunal de Justicia de la Unión Europea (Sala Segunda) de 7 de junio de 2018, as. C-671/16, Inter-Environnement Bruxelles ASBL y otros contra Región de Bruselas-Capital, en la que se resolvió una cuestión prejudicial planteada por el Consejo de Estado de Bélgica actuando como Tribunal Supremo de lo Contencioso-Administrativo, y que tuvo de nuevo como objeto la interpretación de la Directiva 2001/42/CE ${ }^{51}$. También en este pronunciamiento se abordó la interpretación de los artículos 2.a) y 3 de esta directiva, en este caso en relación a la validez del Decreto del gobierno regional por el que se aprobó el reglamento regional de urbanismo zonal y la composición del expediente de solicitud de

\footnotetext{
${ }^{48}$ Véase párrafo 33.

${ }^{49}$ Véase párrafo 34.

${ }^{50}$ Véanse párrafos 65 y 66.

${ }^{51}$ Directiva 2001/42/CE del Parlamento Europeo y del Consejo, de 27 de junio de 2001, relativa a la evaluación de los efectos de determinados planes y programas en el medio ambiente, DO, L 197, de 21 de julio de 2001, p. 30.
} 
certificado urbanístico y de licencia urbanística para el perímetro de la rue de la Loi y sus inmediaciones.

El Tribunal valoró el alcance del reglamento regional para considerar que un acto como éste debía considerarse incluido en el concepto de "planes y programas", a lo que añadió que la necesidad de evaluación ambiental que se contempla para estas actuaciones en la Directiva 2001/42/CE no decae por el hecho de que se vayan a realizar otras evaluaciones en otros ámbitos normativos del Derecho de la Unión Europea ${ }^{52}$. En consecuencia, el Tribunal declaró que "un reglamento regional de urbanismo, como el controvertido en el asunto principal, que establece determinadas prescripciones respecto a la realización de proyectos inmobiliarios, está comprendido en el concepto de "planes y programas" que pueden tener efectos significativos en el medio ambiente, en el sentido de dicha Directiva, y tiene por tanto que ser objeto de una evaluación de los efectos medioambientales".

\subsection{Residuos}

En este apartado se incluye la sentencia del Tribunal de Justicia de la Unión Europea (Sala Tercera) de 11 de julio de 2018, as. C-15/17, Bosphorus Queen Shipping Ltd Corp. contra la Rajavartiolaitos, que resolvió una cuestión prejudicial planteada por el Tribunal Supremo de Finlandia, y en la que se cuestionó la interpretación de la Convención de las Naciones Unidas sobre el Derecho del Mar, y de la Directiva 2005/35/CE, relativa a la contaminación procedente de buques y a la introducción de sanciones, incluidas las sanciones penales, para las infracciones de contaminación, en su versión modificada por la Directiva 2009/123/CE ${ }^{53}$. El litigio principal que produjo este pronunciamiento

\footnotetext{
52 Véanse párrafos 59 y 65.

${ }^{53}$ Convención de las Naciones Unidas sobre el Derecho del Mar, firmada en Montego Bay el 10 de diciembre de 1982, Recopilación de Tratados de las Naciones Unidas, vol. 1833, 1834 y 1835, p. 3; Directiva 2005/35/CE del Parlamento Europeo y del Consejo, de 7 de septiembre de 2005, relativa a la contaminación procedente de buques y a la introducción de sanciones, incluidas las sanciones penales, para las infracciones de contaminación, DO, L 255, de 30 de septiembre, de 2005, p. 11; y Directiva 2009/123/CE del Parlamento Europeo y del Consejo, de 21 de octubre de 2009, por la que se modifica la Directiva 2005/35/CE relativa a la contaminación procedente de buques y la introducción de sanciones para las infracciones, DO, L 280, 27 de octubre de 2009, p. 52.
} 
tenía como objeto una multa impuesta a la sociedad propietaria de un buque de carga registrado en Panamá, debido a la descarga de hidrocarburos efectuada en la zona económica exclusiva finlandesa.

En su pronunciamiento, el Tribunal de Justicia recordó que era competente para la interpretación de aquellos acuerdos internacionales en los que la Unión sea parte, de forma que procedió a analizar y exponer la interpretación de diferentes categorías incluidas tanto en normativa internacional como en el derecho derivado de la Unión Europea ${ }^{54}$. En particular, el Tribunal procedió a la interpretación conjunta de los artículos 220.6 de la Convención de las Naciones Unidas sobre el Derecho del Mar, y 7.2 de la Directiva 2005/35/CE, y señaló, en primer término, que "deben interpretarse en el sentido de que las expresiones "prueba objetiva y clara" y "pruebas objetivas claras", empleadas respectivamente en estas disposiciones, se refieren no solo a la comisión de una infracción, sino también a la prueba de las consecuencias de esa infracción”.

Asimismo, el término "costas o [...] intereses conexos" del artículo 220.6, y el término conexo "costa o intereses conexos", del artículo 7.2, "deben interpretarse en el sentido que, en principio, tienen el mismo significado que la expresión "costas o intereses conexos", empleada en el artículo I, apartado 1, y en el artículo II, apartado 4, del Convenio Internacional relativo a la Intervención en Alta Mar en Casos de Accidentes que causen o puedan causar una Contaminación por Hidrocarburos, celebrado en Bruselas el 29 de noviembre de 1969, debiendo puntualizarse que ese artículo 220, apartado 6, se aplica igualmente a los recursos no vivos del mar territorial del Estado ribereño y a cualesquiera recursos de su zona económica exclusiva”. Por otro lado, el análisis de ambos preceptos llevó al Tribunal a declarar que "deben interpretarse en el sentido de que los recursos del mar territorial o de la zona económica exclusiva a los que se hace referencia en estas disposiciones incluyen tanto a las especies capturadas como a las especies vivas asociadas con las especies capturadas o dependientes de ellas, como son las especies de flora y fauna utilizadas como alimento por las especies capturadas".

\footnotetext{
${ }^{54}$ Véanse párrafos 43 y 44.
} 
A lo anterior se añade que el Tribunal declaró que en principio no procedía "tener en cuenta el concepto de "contaminación considerable"' incluida en el 220.5, de la Convención de las Naciones Unidas sobre el Derecho del Mar al aplicar el artículo 220.6, de este tratado y el artículo 7.2, de la Directiva 2005/35, "ni tampoco al apreciar las consecuencias de una infracción, tal y como se definen en dichas disposiciones". El Tribunal señaló, asimismo, los elementos que permitirían apreciar las consecuencias de las infracciones definidas en estos preceptos, y que debían influir las "características geográficas y ecológicas particulares y la vulnerabilidad de la zona del mar Báltico". Por último, precisó que el artículo 1.2 de la Directiva 2005/35, "debe interpretarse en el sentido de que no permite a los Estados miembros imponer medidas más restrictivas, conformes al Derecho internacional, que las establecidas en el artículo 7, apartado 2, de esta Directiva, cuando este sea aplicable, si bien los Estados miembros tienen la facultad de adoptar otras medidas de alcance equivalente a las previstas en dicho artículo 220, apartado 6".

\subsection{Fiscalidad y protección ambiental.}

En este apartado se incluye una sentencia relativa a la interpretación de la libertad de establecimiento, que conlleva una notable transcendencia en relación a la protección ambiental. La sentencia del Tribunal de Justicia de la Unión Europea (Sala Primera) de 26 de abril de 2018, as. C-233/16, Asociación Nacional de Grandes Empresas de Distribución (ANGED) contra Generalitat de Catalunya, resolvió una cuestión prejudicial planteada por el Tribunal Supremo español, y que tuvo como objeto la interpretación de los artículos 49, 54 y 107.1 del Tratado de Funcionamiento de la Unión Europea relativos a la libertad de establecimiento, en relación a la legalidad de un impuesto que gravaba a los grandes establecimientos comerciales situados en la Comunidad Autónoma de Cataluña y cuyo objetivo era compensar su impacto sobre el territorio y el medioambiente.

En su pronunciamiento, el Tribunal consideró, en primer término, que no había elementos que pudieran justificar que el impuesto comprometido resultaba 
discriminatorio por gravar únicamente a grandes superficies ${ }^{55}$. Asimismo, tampoco consideró que pudiera calificarse de ayuda de Estado la exoneración de este impuesto a determinados establecimientos, que o bien no alcanzaban el tamaño de superficie de ventas o que se dedicaban a la venta de determinados productos. En relación a este aspecto, el Tribunal de Justicia recordó que el objetivo del impuesto era contribuir a la protección del medio ambiente y a la ordenación del territorio, y señaló que en relación a estos aspectos la utilización del criterio de la dimensión del establecimiento resultaba relevante y coherente con el fin perseguido y, por tanto, no otorgaba una ventaja selectiva como supondría una ayuda de Estado ${ }^{56}$. Respecto a los establecimientos exonerados por realizar determinadas actividades comerciales, el Tribunal de Justicia aceptó el argumento de la Generalitat de Catalunya en el sentido de que por sus características tienen menos repercusión ambiental, aunque señaló que correspondía al órgano jurisdiccional nacional comprobar que realmente se producía esta circunstancia ${ }^{57}$.

No obstante lo señalado, el Tribunal introdujo una apreciación diferente en relación a los establecimientos comerciales colectivos, respecto a los que no se apreció relación alguna respecto a un diferente impacto ambiental. El impuesto controvertido también incluía una exoneración a estos establecimientos pese a que tuvieran la misma superficie que los que entraban en su ámbito de aplicación, y el Tribunal declaró que en este caso debía interpretarse que esto suponía una ayuda de Estado, y se refirió a la posibilidad de su categorización como ayudas existentes ${ }^{58}$.

\footnotetext{
55 Véase párrafo 32.

${ }^{56}$ Véanse párrafos 52 a 56.

57 Véanse párrafos 59 a 60.

58 Véase párrafo 68.
} 